\title{
Effect of Topical Dexmedetomidine $(0.0055 \%)$ on Intraocular Pressure in Healthy Eyes: A Randomized Controlled Trial
}

\author{
Henry Fakhoury ${ }^{1}$, Youssef Abdelmassih ${ }^{2}$, Sylvain El-Khoury ${ }^{3}$, Mazen Amro ${ }^{4}$, Karen Zaarour ${ }^{5}$, Carole Cherfan ${ }^{6}$, Karim Tomey ${ }^{7}$, \\ Ziad Khoueir ${ }^{8}$
}

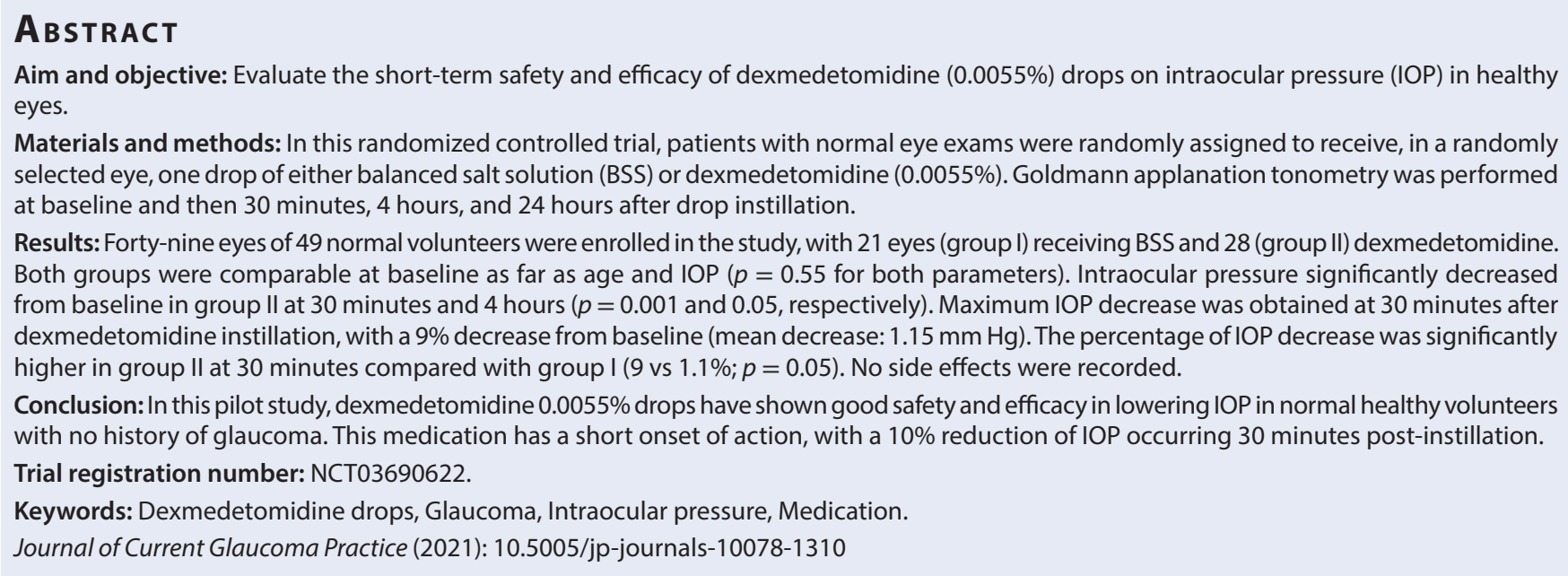

\section{INTRODUCTION}

Glaucoma is the second leading cause of blindness and the leading cause of irreversible blindness worldwide. ${ }^{1}$ It is estimated that glaucoma will be affecting almost 80 million people worldwide by the year $2020 .^{2}$ To date, the only available option to treat glaucoma is through an intraocular pressure (IOP) lowering by medical, laser, or surgical treatment, with topical medical treatment being usually the first-line therapy.

Several drug groups with different mechanisms of action have been proposed to serve this purpose. ${ }^{3}$ However, as the disease advances, medical treatment may become insufficient. Additionally, drug resistance and side effects, such as redness, ocular surface disease, and allergy, are common and may limit the use of some drugs. ${ }^{4,5}$ These restraints often force the physician to keep changing medications, which emphasizes the clinical importance of having a wide armamentarium of drugs available for the treatment of glaucoma.

Dexmedetomidine (Precedex, Pfizer, New York, USA) is a selective $a-2$ agonist with a $1,500 \times$ greater affinity to $a-2$ adrenergic receptors than to a-1 receptors. ${ }^{6}$ It is commonly used as an anesthetic drug due to its sedative and analgesic effects. However, the activation of a-2 receptors is also known to lead to a decrease in IOP. This effect is due to a reduction in aqueous humor production and increased uveoscleral outflow, ${ }^{7-9}$ and was noted in cases where dexmedetomidine was injected intravenously. ${ }^{10,11}$ In addition, the activation of a-2 receptors is known to have a vasoconstricting
${ }^{1}$ Anesthesia Department, Lebanese University, Faculty of Medicine, Beirut, Lebanon; Anesthesia Department, Beirut Eye and ENT Specialist Hospital, Beirut, Lebanon

${ }^{2}$ Ophthalmology Department, Beirut Eye and ENT Specialist Hospital, Beirut, Lebanon; Ophthalmology Department, Saint-Joseph University, Faculty of Medicine, Beirut, Lebanon; Retina Department, Fondation Ophtalmologique Adolphe de Rothschild, Service du Dr Caputo, Paris, France

${ }^{3}$ Ophthalmology Department, Beirut Eye and ENT Specialist Hospital, Beirut, Lebanon; Retina Department, Fondation Ophtalmologique Adolphe de Rothschild, Service du Dr Caputo, Paris, France

4,6Ophthalmology Department, Lebanese University, Faculty of Medicine, Beirut, Lebanon; Ophthamology Department, Beirut Eye and ENT Specialist Hospital, Beirut, Lebanon

${ }^{5}$ Ophthalmology Department, Beirut Eye and ENT Specialist Hospital, Beirut, Lebanon; Ophthalmology Department, Saint-Joseph University, Faculty of Medicine, Beirut, Lebanon

${ }^{7}$ Glaucoma Department, Beirut Eye and ENT Specialist Hospital, Beirut, Lebanon; Ophthalmology Department, Lebanese American University, Gilbert and Rose-Mary Chagoury School of Medicine, Byblos, Lebanon ${ }^{8}$ Ophthalmology Department, Saint-Joseph University, Faculty of Medicine, Beirut, Lebanon; Glaucoma Department, Beirut Eye and ENT Specialist Hospital, Beirut, Lebanon; Ophthalmology Department, Mayo Clinic, Jacksonville, Florida, USA

Corresponding Author: Ziad Khoueir, Ophthalmology Department, Saint-Joseph University, Faculty of Medicine, Beirut, Lebanon; Glaucoma Department, Beirut Eye and ENT Specialist Hospital, Beirut,

(0) Jaypee Brothers Medical Publishers. 2021 Open Access This article is distributed under the terms of the Creative Commons Attribution 4.0 International License (https://creativecommons.org/licenses/by-nc/4.0/), which permits unrestricted use, distribution, and non-commercial reproduction in any medium, provided you give appropriate credit to the original author(s) and the source, provide a link to the Creative Commons license, and indicate if changes were made. The Creative Commons Public Domain Dedication waiver (http://creativecommons.org/publicdomain/zero/1.0/) applies to the data made available in this article, unless otherwise stated. 
effect, which reduces hyperemia, a common side-effect of antiglaucoma treatment. ${ }^{12}$

A study performed on rabbits demonstrated a promising effect of dexmedetomidine eye drops as far as lowering IOP..$^{13} \mathrm{~A}$ more recent study using dexmedetomidine $(50 \mu \mathrm{g})$ with the peribulbar block before cataract surgery demonstrated an IOP decrease similar to IV dexmedetomidine administration and greater than a peribulbar block without dexmedetomidine. $^{14}$

Topical $0.0055 \%$ dexmedetomidine has been reported to cause an IOP reduction of $20-44 \%$ in four healthy subjects. ${ }^{15}$

The purpose of this double-masked randomized controlled trial study is to evaluate the effect of topical dexmedetomidine eye drops on the IOP of healthy eyes.

\section{Materials and Methods}

In this prospective double-masked randomized controlled trial, we included healthy volunteers with no history of glaucoma. Subjects were examined at the Beirut Eye and ENT Specialist Hospital between December 2017 and January 2018. The use of topical dexmedetomidine was off-label. The protocol of the study was reviewed and approved by the institutional review board and complied with the tenets of Helsinki. The study is registered with Clinicaltrials.gov (identifier, NCT03690622). ${ }^{16}$

Volunteers were recruited among hospital staff and patients. Subject exclusion criteria were a history of glaucoma, IOP $>24 \mathrm{~mm}$ $\mathrm{Hg}$ at baseline, an abnormal optic nerve on fundus examination, keratoconus, corneal graft, corneal edema, or a history of refractive surgery. All volunteers were required to sign an informed consent explaining the nature of the study as well as the potential side effects of the drug used.

With a power of $85 \%$, an estimated difference of $20 \%$ between dexmedetomidine and placebo, and a standard deviation of $2.6 \mathrm{~mm}$ $\mathrm{Hg}$ for IOP in the normal population, the number of eyes needed to be included in the trial was calculated to be 40 for a two-sided test. ${ }^{17}$

The eye drops were prepared on the morning of each recruitment day, and the bottles were stored in a refrigerator for a maximum time of 24 hours. Every day, two bottles labeled "1" and " 2 " were handed to the investigators. One of the bottles contained a balanced saline solution while the other one contained a solution of $0.0055 \%$ of dexmedetomidine-hydrochloride. For every examination day, the labeling of the bottles was carried out in a randomized fashion, and the pharmacist noted the corresponding content on a sheet of paper, which was not accessible to the investigator.

Furthermore, subjects were randomly assigned to receive, in a randomly selected eye, either a drop of bottle " 1 " or a drop of bottle " 2 ". Patients and investigators were therefore blinded to the content of the eye drop bottles.

All recruited volunteers were examined by slit-lamp, with a non-dilated fundus examination of both eyes before being included in the study. Goldmann applanation tonometry of both eyes was performed at presentation, 30 minutes, 4 hours, and 24 hours after instillation of the eye drop as well as evaluation of conjunctival hyperemia. Heart rate and blood pressure were measured for all patients at the same follow-up times and patients were asked about nausea, vomiting, lypothemia, syncope, dry mouth, and palpitation.

\section{Preparation of Eye Drops}

One milliliter of dexmedetomidine- $\mathrm{HCl}$ (Precedex 0.01\% Pfizer, New York, USA) was diluted in $0.8 \mathrm{~mL}$ of balanced salt solution (BSS) to
Lebanon; Ophthalmology Department, Mayo Clinic, Jacksonville, Florida, USA, Phone: +00961 1397 009, e-mail: ziad@khoueir.com

How to cite this article: Fakhoury $\mathrm{H}$, Abdelmassih $\mathrm{Y}$, El-Khoury $\mathrm{S}$, et al. The Effect of Topical Dexmedetomidine $(0.0055 \%)$ on Intraocular Pressure in Healthy Eyes: A Randomized Controlled Trial. J Curr Glaucoma Pract 2021;15(2):58-63.

Source of support: Nil

Conflict of interest: None

obtain $1.8 \mathrm{~mL}$ of $0.0055 \%$ dexmedetomidine. The solution was then poured into a sterile eye drop container. The placebo bottle contained $2 \mathrm{~mL}$ of BSS and was stored in an identical container. ${ }^{15}$

\section{Statistical Analysis}

Data were collected and statistically analyzed using SPSS (version 22.0, Inc., Chicago, IL, USA). Descriptive statistics were reported as mean \pm standard deviation for continuous variables. Independent and paired sample $t$-test was used to compare the different continuous parameters. A $p$ value of $<0.05$ or less was considered to be statistically significant.

\section{Results}

A total of 49 volunteers were included in the study, with 21 receiving placebo (group I) and 28 receiving dexmedetomidine $0.0055 \%$ solution (group II) (Flowchart 1). Mean age was similar in both groups, with $60.4 \pm 18.2$ years (range: $24-80$ ) in group I and $64 \pm$ 19.8 years (range: $18-90)$ in group II ( $p=0.55)$. Baseline IOP was 14.2 $\pm 3.5 \mathrm{~mm} \mathrm{Hg}$ (range: 9-23) in the eyes receiving the placebo solution and $13.6 \pm 3.6 \mathrm{~mm} \mathrm{Hg}$ (range: 6-19) in the dexmedetomidine group $(p=0.55)$ (Table 1).

\section{Eyes Receiving Drops}

For group I, IOP did not significantly change in comparison to baseline and was measured to be $14.1 \pm 4.3$ at 30 minutes ( $p=$ $0.84), 14.4 \pm 3.2 \mathrm{~mm} \mathrm{Hg}$ at 4 hours $(p=0.13)$, and $13.5 \pm 3.6 \mathrm{~mm} \mathrm{Hg}$ $(p=0.26)$ at 1 day (Table 2 and Fig. 1). Intraocular pressure change was $-1.1 \%$ at 30 minutes, $+4.7 \%$ at 4 hours, and $-2.5 \%$ at 24 hours (Table 3).

In group II, however, at 30 minutes, 4 hours, and 24 hours, IOP decreased to $12.4 \pm 3.9,12.5 \pm 3.9$, and $12.8 \pm 3.5$, respectively, with the difference from baseline being statistically significant only at 30 minutes $(p=0.001)$ and at 4 hours $(p=0.05)$, but not at 24 hours $(p=0.09$ ) (Table 2 and Fig. 1). Intraocular pressure, in comparison to baseline, decreased by $9 \%$ at 30 minutes, $8.2 \%$ at 4 hours, and $4.6 \%$ at 24 hours (Table 3). An average decrease in IOP of $1.15 \mathrm{~mm} \mathrm{Hg}$ was noted at the 30 minutes measurement (range: -1 to $5 \mathrm{~mm} \mathrm{Hg}$ ).

Intraocular pressure difference between both groups increased from baseline over time without reaching statistical significance. The mean difference was $0.6 \mathrm{~mm} \mathrm{Hg}$ at baseline, $1.7 \mathrm{~mm} \mathrm{Hg}$ at 30 minutes ( $p=0.17), 1.9 \mathrm{~mm} \mathrm{Hg}$ at 4 hours $(p=0.15)$ and $0.7 \mathrm{~mm}$ $\mathrm{Hg}$ at 24 hours $(p=0.57)$ (Table 1). A significant difference in the percentage of IOP change between groups was present only at 30 minutes ( $9 \%$ for group II vs $1.1 \%$ for group I, $p=0.05$ ) (Table 3 ). Figure 2 represents a scatter plot comparing IOP in $\mathrm{mm} \mathrm{Hg}$ at baseline to IOP at the 30-minute follow-up using BSS eye drop and dexmedetomidine eye drop, respectively.

\section{Fellow Eyes}

While comparing the effect of drops between the eye receiving the drop and the fellow eye, the difference was not statistically 
significant in group I at all measurement times. In group II, however, the IOP was significantly lower at 4 hours in eyes receiving dexmedetomidine compared with fellow eyes (12.5 \pm 3.85 vs $14.5 \pm 3.38 \mathrm{~mm} \mathrm{Hg}, p=0.009$ ) (Table 1).

\section{Side Effects}

No ocular side effects were noted in any patient. No systemic side effects such as bradycardia, hypotension, or syncope occurred in any patient.

\section{Discussion}

This prospective randomized controlled trial studied the effect of instilling one drop of dexmedetomidine $0.0055 \%$ (off-label use) on the IOP in eyes with no history of glaucoma or elevated IOP and compared it to sham treatment. Intraocular pressure significantly decreased from baseline at 30 minutes and 4 hours in the dexmedetomidine group. However, while comparing both groups, the only significant difference was found at 30 minutes, where the IOP in group II decreased by 9 vs $1.1 \%$ in group I $(p=0.05)$.

Flowchart 1: Flow diagram for subjects included in the study

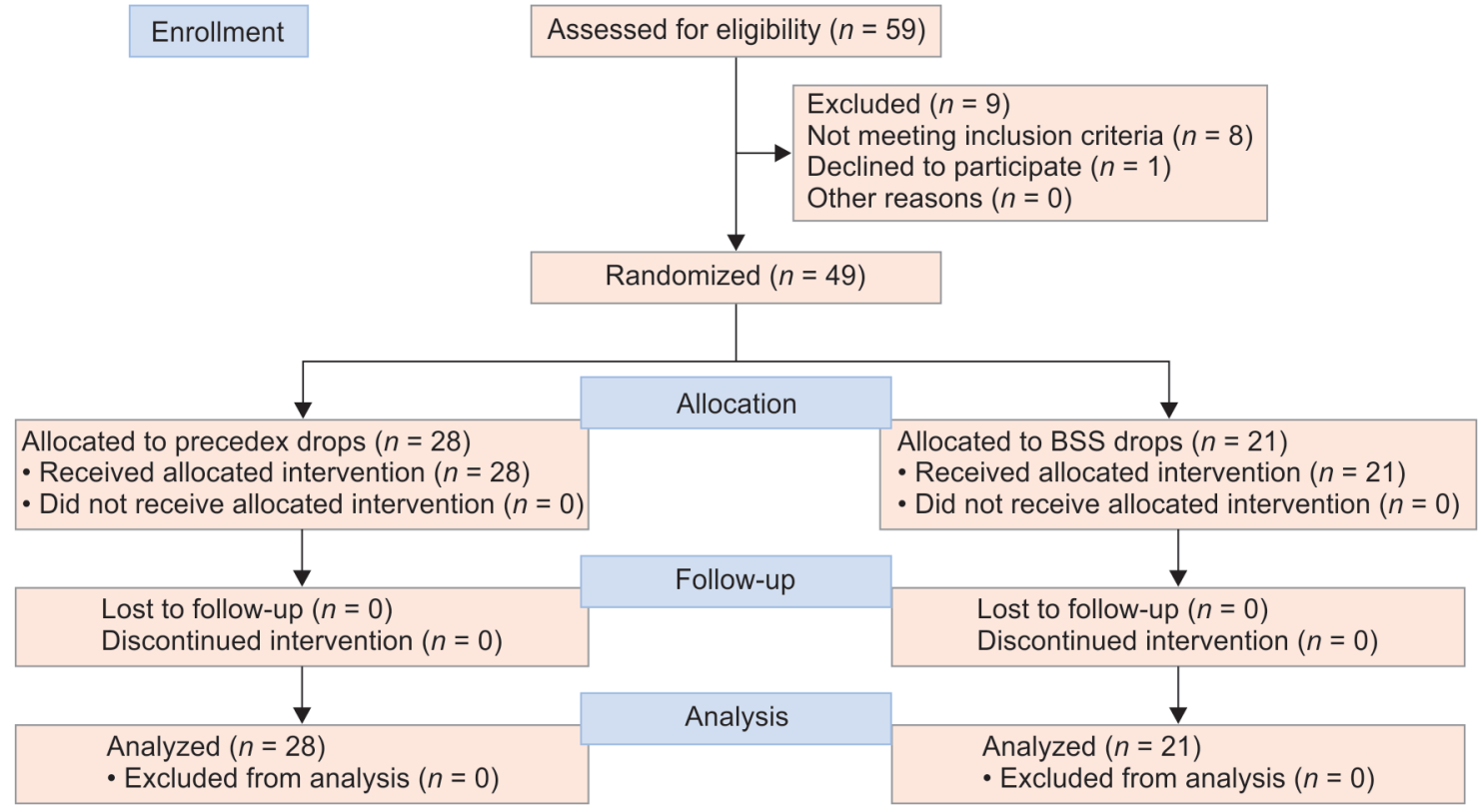

Table 1: Comparison between treated eyes and fellow eyes. Baseline characteristics of all enrolled patients and IOP measurements at different times for eyes receiving drops and fellow eyes. $p$ values for IOP differences between the two groups and between eyes receiving the drop and fellow eye are also reported

\begin{tabular}{|c|c|c|c|}
\hline Baseline characteristics & BSS & Dexmedetomidine & pvalue \\
\hline Number of patients & 21 & 28 & \\
\hline Mean age \pm SD (range) & $60.4 \pm 18.2(24-80)$ & $64.0 \pm 19.8(18-90)$ & 0.55 \\
\hline \multicolumn{4}{|c|}{ Mean IOP at baseline \pm SD (range) } \\
\hline Eyes with drops & $14.2 \pm 3.47(9-23)$ & $13.6 \pm 3.58(6-19)$ & 0.55 \\
\hline Fellow eye & $14.1 \pm 4.86(8-23)$ & $13.6 \pm 3.65(4-19)$ & 0.71 \\
\hline$p$ value & 0.83 & 0.72 & \\
\hline \multicolumn{4}{|c|}{ Mean IOP at 30 minutes \pm SD (range) } \\
\hline Eyes with drops & $14.1 \pm 4.3(9-23)$ & $12.4 \pm 3.9(5-19)$ & 0.17 \\
\hline Fellow eye & $13.9 \pm 5.08(5-23)$ & $13.4 \pm 4.2(2-19)$ & 0.75 \\
\hline$p$ value & 0.74 & 0.17 & \\
\hline \multicolumn{4}{|c|}{ Mean IOP at 4 hours \pm SD (range) } \\
\hline Eyes with drops & $14.4 \pm 3.20(9-20)$ & $12.5 \pm 3.85(6-19)$ & 0.15 \\
\hline Fellow eye & $15.0 \pm 6.36(8-25)$ & $14.5 \pm 3.38(8-19)$ & 0.82 \\
\hline$p$ value & 0.53 & $0.009 *$ & \\
\hline \multicolumn{4}{|c|}{ Mean IOP at 24 hours \pm SD (range) } \\
\hline Eyes with drops & $13.5 \pm 3.64(10-23)$ & $12.8 \pm 3.50(5-18)$ & 0.57 \\
\hline Fellow eye & $14.3 \pm 5.22(8-25)$ & $13.3 \pm 2.75(7-18)$ & 0.53 \\
\hline$p$ value & 0.44 & 0.12 & \\
\hline
\end{tabular}

*Statistical significance $p \leq 0.05$

BSS, balanced salt solution; IOP, intraocular pressure; SD, standard deviation 
Table 2: Comparison of IOP for the same eye at different follow-up times. All the values were compared to baseline IOP

\begin{tabular}{lllll}
\hline & Baseline & 30 minutes & 4 hours & 24 hours \\
\hline BSS & & & & \\
$\quad$ Eyes with drops & $14.2 \pm 3.47$ & $14.1 \pm 4.32(0.84)$ & $14.4 \pm 3.20(0.13)$ & $13.5 \pm 3.64(0.26)$ \\
$\quad$ Fellow eye & $14.1 \pm 4.86$ & $13.9 \pm 5.08(0.70)$ & $15.0 \pm 6.36(0.45)$ & $14.3 \pm 5.22(0.80)$ \\
$\quad$ & & & \\
$\quad$ Dexmedetomidine & & & $12.5 \pm 3.85(0.049)^{*}$ & $12.8 \pm 3.50(0.09)$ \\
$\quad$ Eyes with drops & $13.6 \pm 3.58$ & $12.4 \pm 3.88(0.001)^{*}$ & $14.5 \pm 3.38(0.44)$ & $13.3 \pm 2.75(0.20)$ \\
\hline
\end{tabular}

*Statistical significance $p \leq 0.05$

$\mathrm{BSS}$, balanced salt solution; IOP, intraocular pressure. IOP variation at different follow-ups
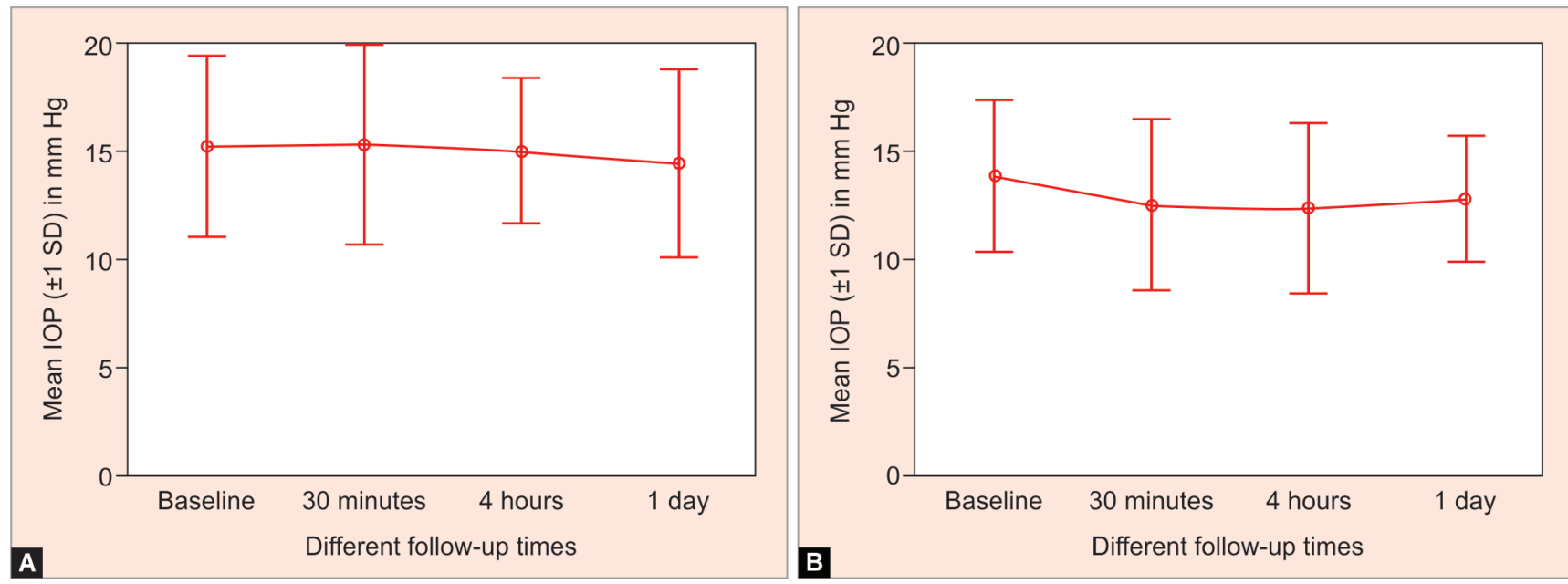

Figs $1 \mathrm{~A}$ and $\mathrm{B}$ : (A) Mean IOP in $\mathrm{mm} \mathrm{Hg}( \pm 1 \mathrm{SD})$ progression curve using BSS over the consecutive follow-up periods; (B) Mean IOP in $\mathrm{mm}$ Hg ( \pm 1 $\mathrm{SD}$ ) progression curve using dexmedetomidine eye drop over the consecutive follow-up periods. BSS, balanced salt solution; IOP, Intraocular pressure; SD, standard deviation

Table 3: Percentage of IOP change from baseline. $p$ values for a percentage of IOP change difference between the two groups and between the eye receiving the drop and the fellow eye are also reported

\begin{tabular}{lccc}
\hline & \multicolumn{3}{c}{ Percentage of IOP change in $\% \pm S D$} \\
\cline { 2 - 4 } & BSS & Dexmedetomidine & $p$ value \\
\hline 30 minutes & $1.1 \pm 14.4$ & $9.0 \pm 12.4$ & $0.05^{*}$ \\
$\quad$ Eyes with drops & $0.9 \pm 15.1$ & $1.5 \pm 20.1$ & 0.90 \\
$\quad$ Fellow eye & 0.90 & 0.16 & \\
$\quad p$ value & & & \\
4 hours & $4.7 \pm 14.1$ & $8.2 \pm 17.6$ & 0.55 \\
$\quad$ Eyes with drops & $-8.6 \pm 31.8$ & $-3.4 \pm 14.3$ & \\
$\quad$ Fellow eye & 0.098 & $0.048^{*}$ & 0.65 \\
$\quad p$ value & & & 0.67 \\
24 hours & $2.5 \pm 13.2$ & $4.6 \pm 13.9$ & \\
$\quad$ Eyes with drops & $1.0 \pm 18.8$ & $4.2 \pm 22.6$ & \\
$\quad$ Fellow eye & 0.65 & 0.89 & \\
$p$ value & & &
\end{tabular}

*Statistical significance $p \leq 0.05$

BSS, balanced salt solution; IOP, intraocular pressure

In our study, 30 minutes was chosen to detect an early effect of one drop of dexmedetomidine and 4 hours represented the time at which maximum effect was reported in the patent. ${ }^{15}$ In the patent, a small experiment on four healthy subjects was performed, resulting in an IOP peak reduction of 40-44\% within 2-5 hours and a 20\% IOP reduction still present 24 hours after instillation. Side effects, such as dry mouth and sedation, were not reported for concentrations $<0.013 \%$. The onset of action was found to be within 1 hour after 

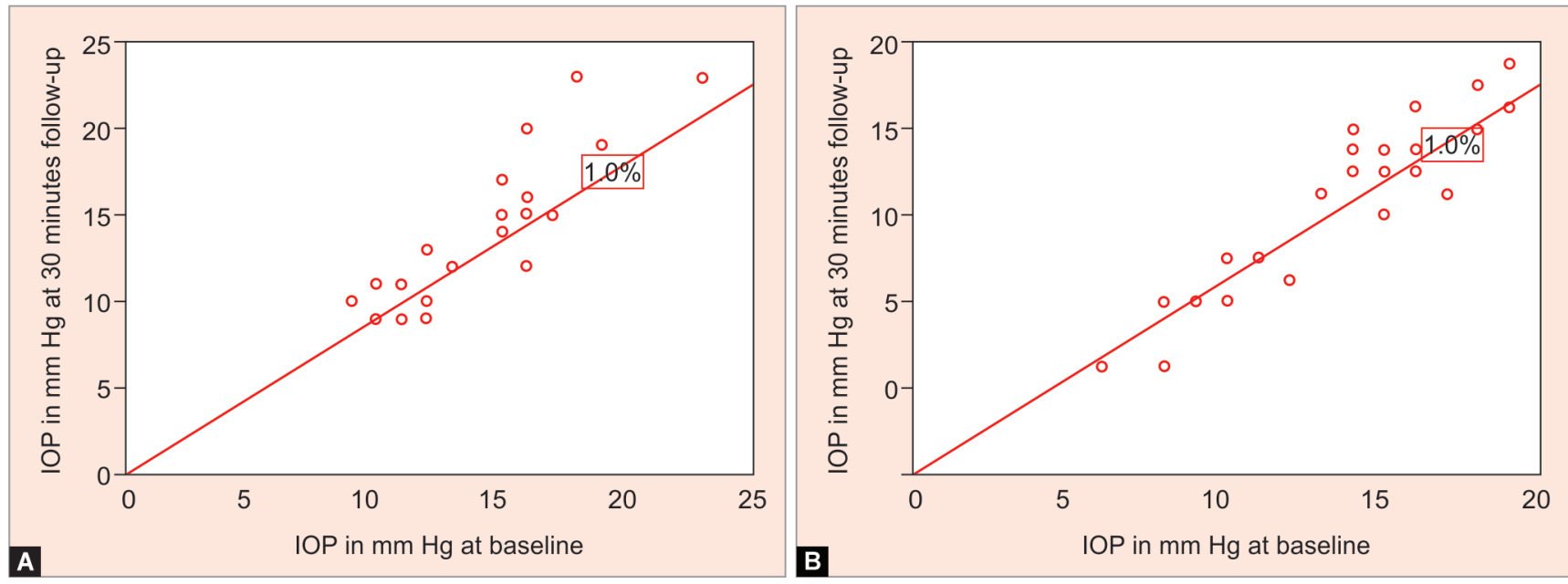

Figs $2 \mathrm{~A}$ and B: (A) Scatter plot comparing IOP in mm Hg at baseline to IOP at the 30-minute follow-up using BSS eye drop; (B) Scatter plot comparing IOP in $\mathrm{mm} \mathrm{Hg}$ at baseline to IOP at the 30-minute follow-up using dexmedetomidine eye drop. The $X$-axis represents baseline IOP and $Y$-axis represents IOP at the 30-minute follow-up. Eyes on or below the cutoff line had a decrease in IOP of at least 10\% and were considered a success. BSS, balanced salt solution; IOP, intraocular pressure

instillation of the eye drop and the half-life was found to be around 2-3 hours, with a maximum effect occurring after around 5 hours. In our study, the decrease in IOP after one dexmedetomidine drop peaked in the early phase after instillation with a maximum decrease of almost 10\% (Table 3 and Fig. 1). This decrease was found to be statistically significant but it was lower than the $40 \%$ that was described in the patent. ${ }^{15}$ It is noteworthy that we used the same concentration in our study like the one that was investigated in the patent. Therefore, it would be possible to think that a higher concentration of dexmedetomidine might be necessary to achieve a stronger decrease of IOP. Another explanation could be that the solvent used for dexmedetomidine dissolution in our study was BSS in an attempt to produce less irritation on the eyes. The solvent and even the $\mathrm{pH}$ of the solution may influence the efficacy of the drug and its intraocular penetration. For example, it has been shown that by adding benzalkonium chloride as a preservative or changing the solution $\mathrm{pH}$ drug penetration and efficacy could be improved. ${ }^{18-20}$

The effect of dexmedetomidine drop is evaluated in normal patients with normal IOP and no history of glaucoma. The effect of dexmedetomidine, or any glaucoma medication, might be more pronounced if it were to be used on patients with an elevated IOP. ${ }^{21,22}$ Furthermore, the study consisted only of the instillation of one drop of dexmedetomidine rather than initiating a full treatment. The IOP-lowering effect of dexmedetomidine eye drop might be more pronounced if used as a chronic treatment, as all known glaucoma drugs usually require at least 1-2 weeks before they reach their full potency. ${ }^{23}$

The relatively short onset of action of dexmedetomidine as shown in our results highlights its potential use to prevent IOP spikes after laser procedures, such as selective laser trabeculoplasty, YAG laser capsulotomy, and peripheral iridotomy. Similar results and IOP curves were obtained in studies that reported the effects of anti-glaucoma drugs, especially a-2 agonists, on IOP after laser treatment. ${ }^{24-27}$ Barnes et al., who reviewed 56 eyes of 41 patients undergoing anterior segment laser, reported a maximum IOP decrease of around $2.3 \mathrm{~mm} \mathrm{Hg}$ up to 4 hours after instillation of either brimonidine $0.2 \%$ or apraclonidine $1.0 \% .^{26}$

No systemic side effects of the drug were reported after the instillation of only one drop of dexmedetomidine, an observation that was also confirmed by the absence of any effect on the fellow eye of patients in group II. Our study illustrates the effect of the instillation of only one drop of $0.0055 \%$ dexmedetomidine in normal eyes. Further studies that include uncontrolled glaucoma patients who are treated for longer periods of time and at concentrations higher than $0.0055 \%$ may be in order, especially that the patent did not show side effects at a concentration lower than $0.013 \%$.

In conclusion, dexmedetomidine $0.0055 \%$ is a safe drug for topical use with no systemic or ocular side effects recorded. It has a fast onset of action with a maximum effect occurring before 4 hours after use and reaching a 10\% decrease of IOP at 30 minutes in normal eyes without a history of glaucoma and after only one eye drop. More studies are needed to evaluate the effect of different solvents and concentrations, as well as to investigate the long-term IOP lowering effect of this drug in glaucoma patients.

\section{References}

1. Tham Y-C, Li X, Wong TY, et al. Global prevalence of glaucoma and projections of glaucoma burden through 2040: a systematic review and meta-analysis. Ophthalmology 2014;121(11):2081-2090. DOI: 10.1016/j.ophtha.2014.05.013.

2. Quigley HA, Broman AT. The number of people with glaucoma worldwide in 2010 and 2020. Br J Ophthalmol 2006;90(3):262-267. DOI: $10.1136 /$ bjo.2005.081224.

3. Tătaru CP, Purcărea VL. Antiglaucoma pharmacotherapy. J Med Life 2012;5(3):247-251.

4. Arita R, Itoh $\mathrm{K}$, Maeda S, et al. Comparison of the long-term effects of various topical antiglaucoma medications on meibomian glands. Cornea 2012;31(11):1229-1234. DOI: 10.1097/ICO.0b013e31823f8e7d.

5. Schuman JS. Antiglaucoma medications: a review of safety and tolerability issues related to their use. Clin Ther 2000;22(2):167-208. DOI: 10.1016/S0149-2918(00)88478-7.

6. Bischoff $P$, Kochs E. Alpha 2-agonists in anesthesia and intensive medicine. Anasthesiologie Intensivmed Notfallmedizin Schmerzther AINS 1993;28(1):2-12. DOI: 10.1055/s-2007-998867.

7. Greenfield DS, Liebmann JM, Ritch R. Brimonidine: a new alpha2-adrenoreceptor agonist for glaucoma treatment. J Glaucoma 1997;6(4):250-258. DOI: 10.1097/00061198-19970800000010. 
8. Toris $\mathrm{CB}$, Gleason $\mathrm{ML}$, Camras $\mathrm{CB}$, et al. Effects of brimonidine on aqueous humor dynamics in human eyes. Arch Ophthalmol Chic III 1960 1995;113(12):1514-1517. DOI: 10.1001/archopht.1995.01100120044006.

9. Toris $C B$, Tafoya $M E$, Camras CB, et al. Effects of apraclonidine on aqueous humor dynamics in human eyes. Ophthalmology 1995;102(3):456-461. DOI: 10.1016/s0161-6420(95)31000-7.

10. Kim NY, Yoo Y-C, Park H, et al. The effect of dexmedetomidine on intraocular pressure increase in patients during robot-assisted laparoscopic radical prostatectomy in the steep Trendelenburg position. J Endourol 2015;29(3):310-316. DOI: 10.1089/end.2014.0381.

11. Zhou C, Zhu Y, Liu Z, et al. The effects of intravenous dexmedetomidine injections on IOP in general anesthesia intubation: a meta-analysis. Biomed Res Int 2017;2017:6186832. DOI: 10.1155/2017/6186832.

12. McAuliffe-Curtin D, Buckley C. Review of alpha adrenoceptor function in the eye. Eye Lond Engl 1989;3(Pt 4):472-476. DOI: 10.1038/ eye.1989.71.

13. Vartiainen J, MacDonald E, Urtti A, et al. Dexmedetomidine-induced ocular hypotension in rabbits with normal or elevated intraocular pressures. Invest Ophthalmol Vis Sci 1992;33(6):2019-2023.

14. Abdelhamid A, Mahmoud A, Abdelhaq M, et al. Dexmedetomidine as an additive to local anesthetics compared with intravenous dexmedetomidine in peribulbar block for cataract surgery. Saudi J Anaesth 2016;10(1):50-54. DOI: 10.4103/1658-354X.169475.

15. Horn G, Compositions and methods for treatment of glaucoma. US8445526 B2, 2013. Available: http://www.google.com.pg/patents/ US8445526.

16. Effect Topical Dexmedetomidine on Intraocular Pressure - Full Text View - ClinicalTrials.gov. [cited 1 Oct 2018]. Available: https:// clinicaltrials.gov/ct2/show/NCT03690622.

17. Chow S, Shao J, Wang H. Sample size calculations in clinical research. 2nd ed., Chapman \& Hall/CRC Biostatistics Series; 2008.

18. Rasmussen CA, Kaufman PL, Kiland JA. Benzalkonium chloride and glaucoma. J Ocul Pharmacol Ther Off J Assoc Ocul Pharmacol Ther 2014;30(2-3):163-169. DOI: 10.1089/jop.2013.0174.
19. Yee RW. The effect of drop vehicle on the efficacy and side effects of topical glaucoma therapy: a review. Curr Opin Ophthalmol 2007;18(2):134-139. DOI: 10.1097/ICU.0b013e328089f1c8.

20. Cantor LB. Brimonidine in the treatment of glaucoma and ocular hypertension. Ther Clin Risk Manag 2006;2(4):337-346. DOI: 10.2147/ tcrm.2006.2.4.337.

21. Heijl A, Leske MC, Hyman L, et al. Intraocular pressure reduction with a fixed treatment protocol in the early manifest glaucoma trial. Acta Ophthalmol (Copenh) 2011;89(8):749-754. DOI: 10.1111/j.17553768.2009.01852.x.

22. Ang GS, Kersey JP, Shepstone L, et al. The effect of travoprost on daytime intraocular pressure in normal tension glaucoma: a randomised controlled trial. Br J Ophthalmol 2008;92(8):1129-1133. DOI: 10.1136/bjo.2007.135269.

23. Parikh RS, Parikh SR, Navin S, et al. Practical approach to medical management of glaucoma. Indian J Ophthalmol 2008;56(3):223-230. DOI: $10.4103 / 0301-4738.40362$.

24. Sridharrao B, Badrinath SS. Efficacy and safety of apraclonidine in patients undergoing anterior segment laser surgery. $\mathrm{Br}$ J Ophthalmol 1989;73(11):884-887. DOI: 10.1136/bjo.73. 11.884 .

25. Chevrier RL, Assalian A, Duperré J, et al. Apraclonidine $0.5 \%$ versus brimonidine $0.2 \%$ for the control of intraocular pressure elevation following anterior segment laser procedures. Ophthalmic Surg Lasers 1999;30(3):199-204. DOI: 10.3928/1542-8877-19990301-08.

26. Barnes SD, Campagna JA, Dirks MS, et al. Control of intraocular pressure elevations after argon laser trabeculoplasty: comparison of brimonidine $0.2 \%$ to apraclonidine $1.0 \%$. Ophthalmology 1999;106(10):2033-2037. DOI: 10.1016/S0161-6420(99) 90420-7.

27. Zhang L, Weizer JS, Musch DC. Perioperative medications for preventing temporarily increased intraocular pressure after laser trabeculoplasty. Cochrane Database Syst Rev 2017;2:CD010746. DOI: 10.1002/14651858.CD010746.pub2. 